\title{
Positive solutions of boundary value problem for singular positone and semi-positone third-order difference equations
}

\author{
Chengjun Yuan ${ }^{1,2^{*}}$, Gongqi Gai ${ }^{2}$ and Yunhui $\mathrm{Li}^{2}$
}

*Correspondence: ycj7102@163.
com
'School of Mathematics and
Statistics, Northeast Normal
University, Changchun, 130024,
People's Republic of China
Full list of author information is
available at the end of the article

\section{Abstract}

This article studies the boundary value problems for the third-order nonlinear singular difference equations

$$
\Delta^{3} u(i-2)+\lambda a(i) f(i, u(i))=0, \quad i \in[2, T+2],
$$

satisfying five kinds of different boundary value conditions. This article shows the existence of positive solutions for positone and semi-positone type. The nonlinear term may be singular. Two examples are also given to illustrate the main results. The arguments are based upon fixed point theorems in a cone.

MSC [2008]: 34B15; 39A10.

Keywords: positive solution, positone and semi-positone boundary value problem, singular difference equation, fixed point theorem

\section{Introduction}

In this article, we consider the following dynamic equations:

$$
\Delta^{3} u(i-2)+\lambda a(i) f(i, u(i))=0, \quad i \in[2, T+2],
$$

satisfying one of the following boundary value conditions:

$$
\begin{aligned}
& u(0)=u(1)=u(T+3)=0, \\
& u(0)=u(1)=\Delta u(T+2)=0, \\
& u(0)=u(1)=\Delta^{2} u(T+1)=0, \\
& u(0)=\Delta^{2} u(0)=\Delta u(T+2)=0, \\
& \Delta u(0)=\Delta^{2} u(0)=u(T+3)=0,
\end{aligned}
$$

where $a \in C([2, T+2],(0,+\infty))$.

The existence of positive solutions for nonlinear boundary value problems of difference equation have been studied by several authors. We refer the reader to [1-20] and references therein. In [16], the authors studied the following boundary value problem:

\section{空

(c) 2011 Yuan et al; licensee Springer. This is an Open Access article distributed under the terms of the Creative Commons Attribution License (http://creativecommons.org/licenses/by/2.0), which permits unrestricted use, distribution, and reproduction in any medium, provided the original work is properly cited. 


$$
\Delta^{3} u(i-2)+a(i) f(i, u(i))=0, \quad i \in[2, T+2]
$$

satisfying one of the boundary value conditions $(k)(k=2,3, \ldots, 6)$ with no singularity. The Green functions are constructed carefully, and some verifiable criteria for the existence of at least one positive solution and two positive solutions are obtained by using fixed point theorem.

Recently, some authors studied semi-positone boundary value problem of difference equations, for instance, see [17-20]

The author [17], studied the following second-order semi-positone boundary value problems:

$$
\left\{\begin{array}{l}
\Delta^{2} u(i-1)+\lambda f(u(i))=0, \quad i \in[1, T] \\
u(0)=u(T+1)=0,
\end{array}\right.
$$

where $\lambda>0$ is a parameter, with no singularity, and where $f(t, u) \geq-M$ with $M$ being a positive constant. They obtained nonexistence and multiplicity results on sublinear nonlinearities and an existence result on superlinear nonlinearities for (8), respectively.

In [18], the authors are concerned with the discrete third-order three-point boundary value problem:

$$
\left\{\begin{array}{l}
\Delta^{3} u(i)=\lambda g(i) f(u(i)), \quad i=0,1, \ldots, n-2, \\
u(0)=\Delta u(p)=\Delta^{2} u(n-1)=0,
\end{array}\right.
$$

where $f:(0, \infty) \rightarrow(0, \infty)$ is a continuous function, and $p$ and $n$ are positive integers. The existence of positive solutions corresponding to the first eigenvalue of the problem is established, and an interval estimate for the first eigenvalue is obtained. In the nonlinear case, sufficient conditions for the existence and nonexistence of positive solutions are obtained.

It is noted that the boundary value problem (1) with boundary value condition $(k)$ can be viewed as the discrete analogue of the following boundary value problems for ordinary differential equation:

$$
u^{(3)}(t)+\lambda a(t) f(t, u(t))=0, \quad t \in(0,1),
$$

respectively satisfying the following boundary value conditions

$$
\begin{aligned}
& u(0)=u^{\prime}(0)=u(1)=0, \\
& u(0)=u^{\prime}(0)=u^{\prime}(1)=0, \\
& u(0)=u^{\prime}(0)=u^{\prime \prime}(1)=0, \\
& u(0)=u^{\prime \prime}(0)=u^{\prime}(1)=0, \\
& u^{\prime}(0)=u^{\prime \prime}(0)=u(1)=0 .
\end{aligned}
$$

In engineering, the equation (10) describes an elastic beam in an equilibrium state both the ends of which are simply supported. 
Motivated by the results above mentioned, we study the boundary value problems (1), in which nonlinear term may be singularity. In this article, we shall prove our two existence results for the problem (1) using Krasnosel'skii's fixed point theorem. This article is organized as follows. In section 2, starting with some preliminary lemmas, we state the Krasnosel'skii's fixed point theorem. In Section 3, we give the sufficient conditions which state the existence of multiple positive solutions to the positone boundary value problem (1). In Section 4, we give the sufficient conditions which state the existence of at least one positive solutions to the semi-positone boundary value problem (1).

\section{Preliminaries}

In this section, we state the preliminary information that we need to prove the main results. From Definition 2.1 in [10], we have the following lemmas.

Lemma 2.1 $u(i)$ is a solution of equation (1) with boundary value condition $(k)$ if only and if

$$
u(i)=\sum_{j=2}^{T+2} G_{k}(i, j) a(j) f(j, u(j)), \quad i \in[0, T+3] ;
$$

where $k=2, \ldots, 6$, and

$$
\begin{aligned}
& G_{2}(i, s)= \begin{cases}\frac{i(i-1)(T+3-j)(T+4-j)}{2(T+3)(T+2)}-\frac{(i-j)(i-j+1)}{2}, & 0 \leq j<i, \\
\frac{i(i-1)(T+3-j)(T+4-j)}{2(T+3)(T+2)}, & i \leq j \leq T+3 ;\end{cases} \\
& G_{3}(i, j)= \begin{cases}\frac{i(i-1)(T+3-j)}{2(T+2)}-\frac{(i-j)(i-j+1)}{2}, & 0 \leq j<i, \\
\frac{i(i-1)(T+3-j)}{2(T+2)}, & i \leq j \leq T+3 ;\end{cases} \\
& G_{4}(i, j)= \begin{cases}\frac{i(i-1)}{2}-\frac{(i-s)(i-j+1)}{2}, & 0 \leq j<i, \\
\frac{i(i-1)}{2}, & i \leq j \leq T+3 ;\end{cases} \\
& G_{5}(i, j)= \begin{cases}i(T+3-j)-\frac{(i-j)(i-j+1)}{2}, & 0 \leq j<i, \\
i(T+3-j), & i \leq j \leq T+3 ;\end{cases} \\
& G_{6}(i, j)= \begin{cases}\frac{(T+3-j)(T+4-j)}{2}-\frac{(i-j)(i-j+1)}{2}, & 0 \leq j<i, \\
\frac{(T+3-j)(T+4-j)}{2}, & i \leq j \leq T+3 .\end{cases}
\end{aligned}
$$

Lemma 2.2 [10] For $k=2, \ldots, 6$, we have the conclusions:

$$
\begin{aligned}
& 0 \leq G_{k}(i, j) \leq g_{k}(j), \quad(i, j) \in[0, T+3] \times[2, T+2], \\
& G_{k}(i, j) \geq M_{k} g_{k}(j), \quad(i, j) \in[2, T+2] \times[2, T+2],
\end{aligned}
$$

where

$$
\begin{array}{ll}
g_{2}(j)=G_{2}(\tau(j), j), & \tau(s)=\left[\frac{4 T^{2}+28 T+48-4 j}{8 T+24-4 j}\right], \quad M_{2}=\frac{2}{(T+1)(T+2)}, \\
g_{3}(j)=\frac{(T+3-j)(j-1)}{2}, & M_{3}=\frac{2}{(T+1)(T+2)}, \\
g_{4}(j)=\frac{(2 T+6-j)(j-1)}{2}, & M_{4}=\frac{1}{(T+1)(T+2)}, \\
g_{5}(j)=\frac{(T+3-j)(T+2+j)}{2}, & M_{5}=\frac{2}{T+2} \\
g_{6}(j)=\frac{(T+3-j)(T+4-j)}{2}, & M_{6}=\frac{2}{(T+2)} .
\end{array}
$$


From Lemma 2.2, it is easy to verify the following lemma.

Lemma 2.3 For $k=2, \ldots, 6$, the Green's function $G_{k}(i, j)$ has properties

$$
\begin{aligned}
& 0<M_{0} h_{k}(i) g_{k}(j) \leq G_{k}(i, j) \leq h_{k}(i), \quad(i, j) \in[0, T+3] \times[2, T+2], \\
& \text { where } M_{0}=\min _{2 \leq k \leq 6}\left\{M_{k}^{*}\right\} \text { and } \\
& h_{2}(i)=i(i-1), \quad M_{2}^{*}=\frac{M_{2}}{(T+3)^{2}} \text {, } \\
& h_{3}(i)=i(i-1), \quad M_{3}^{*}=\frac{M_{3}}{(T+3)^{2}}, \\
& h_{4}(i)=i(i-1), \quad M_{4}^{*}=\frac{M_{4}}{(T+3)^{2}} \text {, } \\
& h_{5}(i)=(T+3) i, \quad M_{5}^{*}=\frac{M_{5}}{T+3}, \\
& h_{6}(i)=(T+4)(T+3-i), \quad M_{6}^{*}=\frac{M_{6}}{T+4} \text {. }
\end{aligned}
$$

For our constructions, we shall consider the Banach space $E=C[0, T+3]$ equipped with the standard norm $\|u\|=\max _{0 \leq i \leq T+3}|u(i)|, u \in E$. We define a cone $P_{k}(k=2, \ldots, 6)$ by

$$
P_{k}=\left\{u \in X \mid u(i) \geq M_{0} h_{k}(i)\|u\|, \quad i \in[0, T+3]\right\} .
$$

We note that $u(i)$ is a solution of (1) with boundary value condition $(k)(k=2, \ldots, 6)$ if and only if

$$
u(i)=\lambda \sum_{j=2}^{T+2} G_{k}(i, j) a(j) f(j, u(j)) .
$$

The following theorems will play major role in our next analysis.

Theorem 2.4 [21] Let $X$ be a Banach space, and let $P \subset X$ be a cone in $X$. Let $\Omega_{1}$, $\Omega_{2}$ be open subsets of $X$ with $0 \in \Omega_{1} \subset \bar{\Omega}_{1} \subset \Omega_{2}$, and let $S: P \rightarrow P$ be a completely continuous operator such that, either

1. $\|S w\| \leq\|w\|, w \in P \cap \partial \Omega_{1},\|S w\| \geq\|w\|, w \in P \cap \partial \Omega_{2}$, or

2. $\|S w\| \geq\|w\|, w \in P \cap \partial \Omega_{1},\|S w\| \leq\|w\| w \in P \cap \partial \Omega_{2}$.

Then $S$ has a fixed point in $P \cap \bar{\Omega}_{2} \backslash \Omega_{1}$.

\section{Singular positone problems}

Theorem 3.1 Let us assume that the following conditions are satisfied,

(H1) $f \in C([2, T+2] \times(0,+\infty),[0,+\infty))$;

(H2) $f(i, u) \leq K(i)(g(u)+h(u))$ on $[2, T+2] \times(0, \infty)$ with $g>0$ continuous and nonincreasing on $(0, \infty), h \geq 0$ continuous on $[0, \infty]$ and $\frac{h}{g}$ non-decreasing on $(0, \infty), \exists K_{0}$ with $g(x y) \leq K_{0} g(x) g(y) \forall x>0, y>0$;

(H3) There exists $[\alpha, \beta] \subset[2, T+2]$ such that $\lim _{u \rightarrow+\infty} \inf \frac{f(i, u)}{u}=+\infty$ for $i \in[\alpha, \beta]$; and

(H4) There exists $\left[\alpha_{1}, \beta_{1}\right] \subset[2,+2]$ such that $\lim _{u \rightarrow 0+} \inf \frac{f(i, u)}{u}=+\infty$ for $i \in\left[\alpha_{1}, \beta_{1}\right]$.

Then for each $r>0$, there exists a positive number $\lambda^{*}$ such that the problem (1) with boundary value condition $(k)(k=2, \ldots, 6)$ has at least two positive solutions for $0<\lambda$ $<\lambda *$. 
Proof. Now, we let $k \in[2,6]$ and define the integral operator $T_{k}: P_{k} \rightarrow E$ by

$$
T_{k} u(i)=\lambda \sum_{j=2}^{T+2} G_{k}(i, j) a(j) f(j, u(j)),
$$

where $P_{k}=\left\{u \in X \mid u(i) \geq M_{0} h_{k}(i)\|u\|, i \in[0, T+3]\right\}$.

It is easy to check that $T_{k}\left(P_{k}\right) \subset P_{k}$. In fact, for each $u \in P_{k}$, we have by Lemma 2.2 that

$$
T_{k} u(i) \leq \lambda \sum_{j=2}^{T+2} g_{k}(j) a(j) f(j, u(j)) .
$$

This implies $\left\|T_{k} u\right\| \leq \lambda \sum_{j=2}^{T+2} g_{k}(j) a(j) f(u(j))$. On the other hand, we have

$$
T_{k} u(i) \geq M_{0} h_{k}(i) \lambda \sum_{j=2}^{T+2} g_{k}(j) a(j) f(j, u(j))
$$

Thus we have $T_{k}\left(P_{k}\right) \geq P_{k}$. In addition, standard argument show that $T_{k}$ is completely continuous.

For any $r>0$ given, and take $\Omega_{r}=\{u \in E \mid\|u\|<r\}$. Choose

$$
\lambda^{*}=\frac{r}{K_{0}^{2} g\left(M_{0}\right) \sum_{j=2}^{T+2} g_{k}(j) a(j) K(j) g\left(h_{k}(j)\right)(g(r)+h(r))} .
$$

For $u \in P \cap \partial \Omega_{r}$. From (H2) and (18), we have

$$
\begin{aligned}
T_{k} u(i) & =\lambda \sum_{j=2}^{T+2} G_{k}(i, j) a(j) f(j, u(j)) \\
& \leq \lambda \sum_{j=2}^{T+2} g_{k}(j) a(j) K(j) g(u)\left(1+\frac{h(u)}{g(u)}\right) \\
& \leq \lambda \sum_{j=2}^{T+2} g_{k}(j) a(j) K(j) g\left(M_{0} h_{k}(j) r\right)\left(1+\frac{h(r)}{g(r)}\right) \\
& \leq \lambda K_{0}^{2} g\left(M_{0}\right) \sum_{j=2}^{T+2} g_{k}(j) a(j) K(j) g\left(h_{k}(j)\right)(g(r)+h(r)) \\
& <r .
\end{aligned}
$$

Thus,

$$
\left\|T_{k} u\right\|<\|u\| \text { for } u \in P_{k} \cap \partial \Omega_{r} .
$$

Further, choose a constant $M_{*}>0$ satisfying that

$$
\lambda M^{*} M_{0} \sigma \max _{0 \leq i \leq T+3}\left\{\sum_{j=\alpha}^{\beta} G_{k}(i, j) a(j)\right\}>1,
$$

where $\sigma=\min _{\alpha \leq i \leq \beta}\left\{h_{k}(i)\right\}$. 
From $\lim _{u \rightarrow+\infty} \inf \frac{f(i, u)}{u}=+\infty$ for $i \in[\alpha, \beta]$, namely (H3), there is a constant $L>r$ such that

$$
f(i, x) \geq M^{*} x, \quad \forall x \geq L, i \in[\alpha, \beta] .
$$

Let $R=r+\frac{L}{M_{0} \sigma}$ and $\Omega_{R}=\{u \in E \mid\|u\|<R\}$. For $u \in P_{k} \cap \partial \Omega_{R}$, we have that

$$
u(i) \geq M_{0} h_{k}(i)\|u\| \geq M_{0} R h_{k}(i) \geq M_{0} R \sigma \geq L, \quad i \in[\alpha, \beta] .
$$

It follows that

$$
f(i, u(i)) \geq M^{*} u(i) \geq M^{*} M_{0} R \sigma, \quad i \in[\alpha, \beta] .
$$

Then, for $u \in P \cap \partial \Omega_{R}$, we have

$$
\begin{aligned}
\left\|T_{k} u\right\| & =\lambda \max _{0 \leq i \leq T+3}\left\{\sum_{j=2}^{T+2} G_{k}(i, j) a(j) f(j, u(j))\right\} \\
& \geq \lambda \max _{0 \leq i \leq T+3}\left\{\sum_{j=\alpha}^{\beta} G_{k}(i, j) a(j) f(j, u(j))\right\} \\
& \geq \lambda \max _{0 \leq i \leq T+3}\left\{\sum_{j=\alpha}^{\beta} G_{k}(i, j) a(j) M^{*} M_{0} R \sigma\right\} \\
& \geq \lambda M^{*} M_{0} R \sigma \max _{0 \leq i \leq T+3}\left\{\sum_{j=\alpha}^{\beta} G_{k}(i, j) a(j)\right\} \\
& \geq R .
\end{aligned}
$$

Therefore, by the first part of the Fixed Point Theorem 2.4, $T_{k}$ has a fixed point $u_{2}$ with $r \leq\left\|u_{2}\right\| \leq R$.

Finally, choose a constant $M_{*}>0$ satisfying that

$$
\lambda M_{*} M_{0} \max _{0 \leq i \leq T+3}\left\{\sum_{j=\alpha_{1}}^{\beta_{1}} G_{k}(i, j) a(j) h_{k}(j)\right\}>1 .
$$

By (H4), i.e., $\lim _{u \rightarrow 0+} \inf \frac{f(i, u)}{u}=+\infty$ for $i \in\left[\alpha_{1}, \beta_{1}\right]$, there is a constant $\delta>0$ and $\delta<r$ such that

$$
f(i, u) \geq M_{*} u, \quad \forall u \leq \delta, i \in\left[\alpha_{1}, \beta_{1}\right] .
$$

Let $r_{*}=\frac{\delta}{2}$ and $\Omega_{r_{*}}=\left\{u \in E \mid\|u\|<r_{*}\right\}$. For $u \in P_{k} \cap \partial \Omega_{r_{*}}$, we have

$$
u(i) \geq M_{0} h_{k}(i)\|u\| \geq M_{0} r_{*} h_{k}(i) .
$$

It follows that

$$
f(i, u(i)) \geq M_{*} u(i) \geq M_{*} M_{0} r_{*} h_{k}(i), \quad i \in\left[\alpha_{1}, \beta_{1}\right] .
$$


Then, for $u \in P_{k} \cap \partial \Omega_{r_{*}}$, we have

$$
\begin{aligned}
\left\|T_{k} u\right\| & =\lambda \max _{0 \leq i \leq T+3}\left\{\sum_{j=2}^{T+2} G_{k}(i, j) a(j) f(j, u(j))\right\} \\
& \geq \lambda \max _{0 \leq i \leq T+3}\left\{\sum_{j=\alpha_{1}}^{\beta_{1}} G_{k}(i, j) a(j) f(j, u(j))\right\} \\
& \geq \lambda \max _{0 \leq i \leq T+3}\left\{\sum_{j=\alpha_{1}}^{\beta_{1}} G_{k}(i, j) a(j) M_{*} M_{0} r_{*} h_{k}(j)\right\} \\
& \geq \lambda M_{*} M_{0} r_{*} \max _{0 \leq i \leq T+3}\left\{\sum_{j=\alpha_{1}}^{\beta_{1}} G_{k}(i, j) a(j) h_{k}(j)\right\} \\
& \geq r_{*} .
\end{aligned}
$$

Therefore, by the first part of the Fixed Point Theorem 2.4, $T_{k}$ has a fixed point $u_{1}$ with $r_{*} \leq\left\|u_{1}\right\| \leq r$. It follows from (19) that $\left\|u_{1}\right\| \neq r$.

Then for each $r>0$, there exists a positive number $\lambda$ * such that the problem (1) with boundary value condition $(k)(k=2, \ldots, 6)$ has at least two positive solutions $u_{n}(n=1$, 2) with $r_{*} \leq\left\|u_{1}\right\|<r \leq\left\|u_{2}\right\| \leq R$ for $0<\lambda<\lambda *$.

This completes the proof of the theorem.

From the proof of Theorem 3.1, we have the following result.

Corollary 3.2 Assume that (C1)-(C2) hold. Further, suppose that (H1)-(H3) are satisfied. Then for each $r>0$, there exists a positive number $\lambda^{*}$ such that the problem (1) with boundary value condition ( $k$ ) has at least one positive solution for $0<\lambda<\lambda *$.

Corollary 3.3 Assume that (C1)-(C2) hold. Further, suppose that (H1)-(H2) and (H4) are satisfied. Then for each $r>0$, there exists a positive number $\lambda^{*}$ such that the problem (1) with boundary value condition ( $k$ ) has at least one positive solution for $0<\lambda<\lambda^{*}$.

Remark Condition (H3) shows that $f$ have the property $\lim _{u \rightarrow+\infty} \inf \frac{f(i, u)}{u}=+\infty$ for $i \in[\alpha$, $\beta]$; condition (H4) shows that $f$ have the property $\lim _{u \rightarrow 0+} \inf \frac{f(i, u)}{u}=+\infty$ for $i \in\left[\alpha_{1}, \beta_{1}\right]$.

Example 3.1 Consider the boundary value problem:

$$
-\Delta^{3} u(i-2)=\lambda a(i)\left(c(i) u^{-a}+d(i) u^{b}\left(\sin ^{2} u+1\right)\right), \quad i \in[2, T+2],
$$

with boundary value condition $(k)$, where $0<a<1<b$ are constants, and

$$
c(i)=\left\{\begin{array}{l}
1, i \in[2, T], \\
0, i \in[T+1, T+2],
\end{array} d(i)=\left\{\begin{array}{l}
0, i \in[2, T-2] \\
1, i \in[T-2, T+2] .
\end{array}\right.\right.
$$

Then for each $r>$, there exists a positive number $\lambda^{*}$ such that the problem (22) has at least two positive solutions for $0<\lambda<\lambda^{*}$.

In fact, it is clear that

$$
f(i, u)=c(i) u^{-a}+d(i) u^{b}\left(\sin ^{2} u+1\right)
$$

and

$$
\begin{aligned}
& \lim _{u \rightarrow 0+} \frac{f(i, u)}{u}=+\infty \text { for }[\alpha, \beta]=[2, T] \subset[2, T+2] \\
& \lim _{u \rightarrow+\infty} \frac{f(i, u)}{u}=+\infty \text { for }\left[\alpha_{1}, \beta_{1}\right]=[T-2, T+2] \subset[2, T+2] .
\end{aligned}
$$


Let $K(i)=1, g(u)=u^{-a}$ and $h(u)=2 u^{b}$, we have

$$
f(i, u) \leq K(i)(g(u)+h(u)), \quad K(i)=1
$$

and $g>0$ continuous and non-increasing on $(0, \infty), h \geq 0$ continuous on $(0, \infty)$ and $\frac{h}{g}=2 u^{a+b}$ non-decreasing on $(0, \infty) ; K_{0}=1$ with $g(x y)=g(x) g(y) \leq K_{0} g(x) g(y) \forall x>0, y$ $>0$;

Then, by Theorem 3.1, for each $r>0$ given, we choose

$$
\lambda^{*}=\frac{M_{0}^{a} r^{1+a}}{\left(1+2 r^{a+b}\right) \sum_{j=2}^{T+2} g_{k}(j) a(j) h_{k}^{-a}(j)},
$$

such that the problem (22) has at least two positive solutions for $0<\lambda<\lambda^{*}$.

\section{Singular semi-positone problems}

Before we prove our next main result, we first state a result.

Lemma 4.1 The difference equation

$$
-\Delta^{3} u(i-2)=\lambda a(i) e(i), \quad i \in[2, T+2],
$$

with boundary value condition $(k)$ has a solution $w$ with $w(t) \leq c_{0} h_{k}(i)$, where $c_{0}=\sum_{j=2}^{T+2} a(j) e(j)$.

In fact, from Lemma 2.1, equation (23) has the solution:

$$
w(t)=\sum_{j=2}^{T+2} G_{k}(i, j) a(j) e(j) .
$$

According to Lemma 2.3, we have

$$
w(t) \leq h_{k}(i) \sum_{j=2}^{T+2} a(j) e(j)=c_{0} h_{k}(i) .
$$

Theorem 4.2 Assume that the following conditions are satisfied:

(B1) $f:[2, T+2] \times(0, \infty) \rightarrow R$ is continuous and there exists a function $e \in C([2, T$ $+2],(0,+\infty))$ with $f(i, u)+e(i) \geq 0$ for $(i, u) \in[2, T+2] \times(0, \infty)$;

(B2) $f^{*}(i, u)=f(i, u)+e(i) \leq K(i)(g(u)+h(u))$ on $[2, T+2] \times(0, \infty)$ with $g>0$ continuous and non-increasing on $(0, \infty), h \geq 0$ continuous on $[0, \infty)$ and $\frac{h}{g}$ non-decreasing on $(0, \infty)$;

(B3) $\exists K_{0}$ with $g(x y) \leq K_{0} g(x) g(y) \forall x>0, y>0$;

(B4) There exists $[\alpha, \beta] \subset[2, T+2]$ such that $\lim _{u \rightarrow+\infty} \inf \frac{f(i, u)}{u}=+\infty$ for $i \in[\alpha, \beta]$.

Then for each $r>0$, there exists a positive number $\lambda^{*}$ such that the problem (1) with boundary value condition $(k)$ has at least one positive solution for $0<\lambda<\lambda^{*}$.

Proof. To show (1) with boundary value condition that $(k)$ has a non-negative solution, we will look at the equation:

$$
-\Delta^{3} \gamma(i-2)=\lambda a(i) f^{*}(i, \gamma(i)-\varphi(i)), \quad i \in[2, T+2],
$$

with boundary value condition $(k)$, where $\phi(i)=\lambda w(i) ; w$ is as in Lemma 4.1. 
We let fixed $k \in[2,6]$. We will show, using Theorem 2.4, that there exists a solution $y$ to $(24)$ with $y(i)>\phi(i)$ for $i \in[2, T+2]$. If this is true, then $u(i)=y(i)-\phi(i)(0 \leq i \leq$ $T+4$ ) is a non-negative solution (positive on $[2, T+2]$ ) of (1), since

$$
\begin{aligned}
-\Delta^{3} u(i-2) & =-\Delta^{3}(y(i-2)-\varphi(i-2)) \\
& =\lambda a(i) f^{*}(i, y(i)-\varphi(i))-\lambda a(i) e(i) \\
& =\lambda a(i)[f(i, y(i)-\varphi(i))+e(i)]-\lambda a(i) e(i) \\
& =\lambda a(i) f(i, y(i)-\varphi(i)) \\
& =\lambda a(i) f(i, u(i)), \quad i \in[0, T+4] .
\end{aligned}
$$

Next let $T_{k}: K \rightarrow E$ be defined by

$$
\left(T_{k} y\right)(i)=\lambda \sum_{j=2}^{T+2} G_{k}(i, j) a(j) f^{*}(s, y(s)-\varphi(s)), \quad 0 \leq i \leq T+3 .
$$

In addition, standard argument shows that $T_{k}\left(P_{k}\right) \subset P_{k}$ and $T_{k}$ is completely continuous.

For any $r>0$ given, let

$$
\Omega_{r}=\{y \in E \mid\|y\|<r\}
$$

and choose

$$
\lambda^{*}=\min \left\{\frac{M_{0} r}{2 c_{0}}, \frac{r}{K_{0}^{2} a_{0}(g(r)+h(r))}\right\},
$$

where $a_{0}=g\left(\frac{M_{0}}{2}\right) \sum_{j=2}^{T+2} g_{k}(j) a(j) K(j) g\left(h_{k}(j)\right)$.

We now show that

$$
\left\|T_{k} y\right\| \leq\|y\| \text { for } y \in P_{k} \cap \partial \Omega_{r} .
$$

To see this, let $y \in P_{k} \cap \partial \Omega_{r}$. Then $\|y\|=r$ and $y(t) \geq M_{0} h_{k}(i) r$ for $i \in[0, T+3]$. For $i \in[0, T+3]$, the Lemma 4.1 and (25) imply that

$$
\gamma(i)-\varphi(i) \geq M_{0} r h_{k}(i)-\lambda c_{0} h_{k}(i) \geq\left(M_{0} r-\lambda c_{0}\right) h_{k}(i) \geq \frac{M_{0} r}{2} h_{k}(i)>0,
$$

and hence, for $i \in[0, T+4]$, we have

$$
\begin{aligned}
\left(T_{k} y\right)(i) & =\lambda \sum_{j=2}^{T+2} G_{k}(i, j) a(j) f^{*}(j, y(j)-\varphi(j)) \\
& \leq \lambda \sum_{j=2}^{T+2} g_{k}(j) a(j) K(j)[g(\gamma(j)-\varphi(j))+h(\gamma(j)-\varphi(j))] \\
& =\lambda \sum_{j=2}^{T+2} g_{k}(j) a(j) K(j) g(\gamma(j)-\varphi(j))\left\{1+\frac{h(\gamma(j)-\varphi(j))}{g(\gamma(j)-\varphi(j))}\right\} \\
& \leq \lambda \sum_{j=2}^{T+2} g_{k}(j) a(j) K(j) g\left(\frac{M_{0} r}{2} h_{k}(j)\right)\left\{1+\frac{h(r)}{g(r)}\right\} d s \\
& \leq \lambda K_{0}^{2} g\left(\frac{M_{0}}{2}\right)(g(r)+h(r)) \sum_{j=2}^{T+2} g_{k}(j) a(j) K(j) g\left(h_{k}(j)\right) \\
& =\lambda K_{0}^{2} a_{0}(g(r)+h(r)) \leq r .
\end{aligned}
$$

This yields $\left\|T_{k} y\right\| \leq r=\|y\|$, and so (26) is satisfied. 
Further, choose a constant $M^{*}>0$ satisfying that

$$
\lambda M^{*} \frac{M_{0}}{2} \sigma \max _{0 \leq i \leq T+3}\left\{\sum_{j=\alpha}^{\beta} G_{k}(i, j) a(j)\right\}>1,
$$

where $\sigma=\min _{\alpha \leq i \leq \beta}\left\{h_{k}(i)\right\}$.

By (B4), there is a constant $L>0$ such that

$$
f^{*}(i, x) \geq M^{*} x, \quad \forall x \geq L, i \in[\alpha, \beta] .
$$

Let $R=r+\frac{2 L}{M_{0} \sigma}$ and $\Omega_{R}=\{y \in E \mid\|y\|<R\}$.

Next we show that

$$
\left\|T_{k} y\right\| \geq\|y\| \quad \text { for } y \in P_{k} \cap \partial \Omega_{R} .
$$

To verify this, let $y \in P_{k} \cap \partial \Omega_{R}$. Then have

$$
\gamma(i)-\varphi(i) \geq M_{0} h_{k}(i)\|y\|-\lambda c_{0} h_{k}(i) \geq \frac{M_{0}}{2} R h_{k}(i) \geq \frac{M_{0}}{2} R \sigma \geq L, \quad i \in[\alpha, \beta] .
$$

It follows that, for $y \in P_{k} \cap \partial \Omega_{R}$, we have

$$
f^{*}(i, y(i)-\varphi(i)) \geq M^{*}(\gamma(i)-\varphi(i)) \geq M^{*} \frac{M_{0}}{2} R \sigma, \quad i \in[\alpha, \beta] .
$$

Then, we have

$$
\begin{aligned}
\left\|T_{k} y\right\| & =\lambda \max _{0 \leq i \leq T+3}\left\{\sum_{j=2}^{T+2} G_{k}(i, j) a(j) f^{*}(j, y(j)-\varphi(j))\right\} \\
& \geq \lambda \max _{0 \leq i \leq T+3}\left\{\sum_{j=\alpha}^{\beta} G_{k}(i, j) a(j) f^{*}(j, y(j)-\varphi(j))\right\} \\
& \geq \lambda \max _{0 \leq i \leq T+3}\left\{\sum_{j=\alpha}^{\beta} G_{k}(i, j) a(j) M^{*} \frac{M_{0}}{2} R \sigma\right\} \\
& \geq \lambda M^{*} \frac{M_{0}}{2} R \sigma \max _{0 \leq i \leq T+3}\left\{\sum_{j=\alpha}^{\beta} G_{k}(i, j) a(j)\right\} \\
& \geq R .
\end{aligned}
$$

This yields $\left\|T_{k} y\right\| \geq\|y\|$, and so (28) holds.

Therefore, by the first part of the Fixed Point Theorem 2.4, $T_{k}$ has a fixed point $y$ with $r \leq\|y\| \leq R$. Since

$$
\gamma(i)-\varphi(i) \geq M_{0} h_{k}(i) r-\lambda c_{0} h_{k}(i) \geq\left(M_{0} r-\lambda c_{0}\right) h_{k}(i)>0, \quad i \in[0, T+3] .
$$

In other words, $u=y-\phi$ is a positive solution of the problem (1) with boundary value condition $(k)$.

This completes the proof of the theorem.

Example 4.1. Consider the boundary value problem:

$$
-\Delta^{3} y(i-2)=\lambda a(i)\left(u^{-a}+c(i) u^{b}-\sin \left(i u+i^{\frac{1}{2}}\right)\right)=0, \quad i \in[2, T+2],
$$


with boundary value condition $(k)$. Where $0<a<1<b$ are constants and

$$
c(i)=\left\{\begin{array}{l}
1, i \in[2, T] \\
0, \text { other. }
\end{array}\right.
$$

Then for each $r>$, there exists a positive number $\lambda^{*}$ such that the problem (29) has at least one positive solution for $0<\lambda<\lambda^{*}$.

To verify this, we will apply Theorem 4.2 (here $\lambda^{*}>0$ as will be chosen later). Let

$$
f(i, u)=u^{-a}+c(i) u^{b}-\sin \left(i u+i^{\frac{1}{2}}\right),
$$

then condition (B1) holds. Next, we let

$$
g(u)=u^{-a}, h(u)=u^{b}+2, K(i)=1, e(i)=1, K_{0}=1 .
$$

It is clear that $0 \leq f(i, u)+e(i) \leq K(i)(g(u)+h(u)), g(x y) \leq K_{0} g(x) g(y)$, and $\lim _{u \rightarrow+\infty} \inf \frac{f(i, u)}{u}=+\infty, i \in[\alpha, \beta]=[2, T] \subset[2, T+2]$ hold, i.e., conditions (B1)-(B4) hold. Thus, all the conditions of Theorem 4.2 are satisfied.

For each $r>$ given, let

$$
\lambda^{*}=\min \left\{\frac{C_{2} r}{2 c_{0} C_{1}}, \frac{r}{K_{0} a_{0}(g(r)+h(r))}\right\},
$$

where

$$
c_{0}=\sum_{j=2}^{T+2} a(j), \quad a_{0}=g\left(\frac{M_{0}}{2}\right) \sum_{j=2}^{T+2} g_{k}(j) a(j) h_{k}^{-\alpha}(j) .
$$

Now Theorem 4.2 guarantees that the above equation has positive solution for $0<\lambda$ $<\lambda^{*}$.

\section{Acknowledgements}

This study is supported by the Scientific Research Fund of Natural Science Foundation of Heilongjiang Province of China (No. A201012), Heilongjiang Provincial Education Department (No. 11553067), a grant from the Ph.D. Programs Foundation of Ministry of Education of China (No. 200918), Key Subject of Chinese Ministry of Education (No. 109051), and NNSF of China (No.10971021)

\section{Author details}

${ }^{1}$ School of Mathematics and Statistics, Northeast Normal University, Changchun, 130024, People's Republic of China

${ }^{2}$ School of Mathematics and computer, Harbin University, Harbin, 150086, People's Republic of China

\section{Authors' contributions}

All authors read and approved the final manuscript.

\section{Competing interests}

The authors declare that they have no competing interests.

Received: 8 January 2011 Accepted: 30 September 2011 Published: 30 September 2011

\section{References}

1. Elaydi, S: An introduction to difference equations, Oth edn. Springer, New York (2005)

2. Agarwal, RP: Difference Equations and Inequalities: Theory, Methods and Applications. Marcel Dekker, New York (1992)

3. Agarwal, RP, Henderson, J: Positive solutions and nonlinear problems for third-order difference equation. Comput Math Appl. 36, 347-355 (1998). doi:10.1016/S0898-1221(98)80035-7

4. Agarwal, RP, O'Regan, D: Discrete conjugate boundaryvalue problems. Appl Math Lett. 37, 39-48 (1999)

5. Avery, Rl, Chyan, CJ, Henderson, J: Twin solutions of boundary value problems for ordinary differential equations and finite difference equations. Comput Math Appl. 42, 695-704 (2001). doi:10.1016/S0898-1221(01)00188-2

6. Eloe, PW: A generalization of concavity for finite differences. J Math Anal Appl. 36, 109-113 (1998)

7. Erbe, LH, Xia, HX, Yu, JS: Global stability of linear non-autonomous delay difference equations. J Difference Equ Appl. 1, 151-161 (1995). doi:10.1080/10236199508808016 
8. Hao, ZC: Nonnegative solutions for semilinear third-order difference equation boundary value problems. Acta Math Sci. $21 \mathrm{~A}, 225-229(2001)$

9. Henderson, J, Wong, PJY: Positive solutions for a system of nonpositive difference equations. Aequationes Math. 62, 249-261 (2001). doi:10.1007/PL00000150

10. Kocic, VL, Ladas, G: Global Behavior of Nonlinear Difference Equations of Higher Order with Applications. Kluwer Academic Publishers, Boston (1993)

11. Hatsunaga, H, Hara, T, Sakata, S: Global attractivity for a nonlinear difference equation with variable delay. Comput Math Appl. 41, 543-551 (2001). doi:10.1016/50898-1221(00)00297-2

12. Yuan, C, Jiang, D, O'Regan, D, Agarwal, RP: Existence and uniqueness of positive solutions of boundary value problems for coupled systems of singular second-order three-point non-linear differential and difference equations. Appl Anal. 87, 921-932 (2008). doi:10.1080/00036810802428979

13. He, Z, Yu, J: On the existence of positive solutions of fourth-order difference equations. Appl Math Comput. 161, 139-148 (2005). doi:10.1016/j.amc.2003.12.016

14. He, T, Su, Y: On discrete fourth-order boundary value problems with three parameters. J Comput Appl Math. 233, 2506-2520 (2010). doi:10.1016/j.cam.2009.10.032

15. Yuan, C, Jiang, D, O'Regan, D: Existence and uniqueness of positive solutions for fourth-order nonlinear singular continuous and discrete boundary value problems. Appl Math Comput. 203, 194-201 (2008). doi:10.1016/j. amc.2008.04.034

16. Yang, C, Weng, P: Green functions and positive solutions for boundary value problems of third-order difference equations. Comput Math Appl. 54, 567-578 (2007). doi:10.1016/j.camwa.2007.01.032

17. Zhang, G, Liu, S: On a class of semipositone discrete boundary value problems. J Math Anal Appl. 325, 175-182 (2007). doi:10.1016/j.jmaa.2005.12.047

18. Ji, J, Yang, B: Positive solutions of discrete third-order three-point right focal boundary value problems. J Difference Equ Appl. 15, 185-195 (2009). doi:10.1080/10236190802008536

19. $\mathrm{Ma}, \mathrm{R}, \mathrm{Gao}, \mathrm{C}$ : Sign-changing solutions of nonlinear boundary value problems of difference equations. Indian J Pure Appl Math. 39, 323-332 (2008)

20. Bai, D, Xu, Y: Positive solutions for semipositone BVPs of second-order difference equations. Indian J Pure Appl Math. $39,59-68(2008)$

21. Guo, D, Lakshmikantham, V: Nonlinear problems in abstract cone. Academic Press, San Diego (1988)

doi:10.1186/1687-1847-2011-38

Cite this article as: Yuan et al:: Positive solutions of boundary value problem for singular positone and semipositone third-order difference equations. Advances in Difference Equations 2011 2011:38.

\section{Submit your manuscript to a SpringerOpen ${ }^{\circ}$ journal and benefit from:}

- Convenient online submission

Rigorous peer review

- Immediate publication on acceptance

- Open access: articles freely available online

- High visibility within the field

- Retaining the copyright to your article

Submit your next manuscript at $\boldsymbol{s p r i n g e r o p e n . c o m ~}$ 